\title{
Impact parameter dependence of the azimuthal asymmetry in lepton pair production in heavy ion collisions
}

\author{
Cong Li, Jian Zhou, and Ya-jin Zhou \\ Key Laboratory of Particle Physics and Particle Irradiation (MOE), \\ Institute of frontier and interdisciplinary science, Shandong University(QingDao), \\ Shandong 266237, China
}

(Received 8 November 2019; accepted 3 February 2020; published 14 February 2020)

\begin{abstract}
We investigate the impact parameter dependence of the $\cos 4 \phi$ azimuthal asymmetry for electromagnetic lepton pair production in heavy ion collisions. The asymmetry induced by linearly polarized coherent photons exhibits strong impact parameter dependence.
\end{abstract}

DOI: 10.1103/PhysRevD.101.034015

\section{INTRODUCTION}

It has long been recognized that dilepton production in heavy ion collisions can serve as a clean and powerful probe for studying the electromagnetic properties of quark gluon plasma (QGP). At low lepton pair transverse $\operatorname{momentum}\left(q_{\perp}\right)$, the dominant production mechanism is the photon-photon fusion process $\left(\gamma \gamma \rightarrow l^{+} l^{-}\right)$which is greatly enhanced due to the large flux of quasi-real photons coherently generated by a fast moving nucleus. The measurements carried out at SPS, RHIC and LHC [1-7] have clearly demonstrated that dilepton production is indeed peaked at very low transverse momentum. The comprehensive understanding of such process is not only important for accurate background estimates in heavy ion collisions, but also interesting from a pure theoretical point of view. For example, there is so far no clear experimental evidence for Coulomb correction effects [8-14]. Moreover, whether the significant $q_{\perp}^{2}$ broadening effects in peripheral and central collisions recently observed by ATLAS and STAR collaborations results from the initial state effect or the various final state effects is still under debate [15-19].

Electromagnetic lepton pair production is usually computed using the equivalent photon approximation introduced by Enrico Fermi (also often referred to as the Weizsäcker-Williams method), in which the photon flux is calculated by treating the fields of charged relativistic heavy ion as external, i.e., classical electromagnetic field. This method has been widely used to compute ultraperipheral heavy ion collisions (UPC) observables [20-25]. In the kinematic regions typically accessible at RHIC and

Published by the American Physical Society under the terms of the Creative Commons Attribution 4.0 International license. Further distribution of this work must maintain attribution to the author(s) and the published article's title, journal citation, and DOI. Funded by SCOAP.
LHC, these associated quasireal photons carry very small longitudinal momentum fraction $(x)$ of each nucleon inside the nucleus. In the small $x$ limit, there is a strong correlation between the photon's polarization tensor and its transverse momentum. In the transverse momentum dependent (TMD) factorization framework, such correlation is characterized by the linearly polarized photon TMD [26]. It can give rise to a $\cos 4 \phi$ azimuthal modulation in the dilepton production cross section, where $\phi$ is the angle between the lepton pair transverse momentum and the individual lepton transverse momentum. We recently computed this $\cos 4 \phi$ asymmetry and found that it is rather sizable [27]. We note that the same observable has been studied in the context of nucleon-nucleon collisions in an earlier work [28].

As a matter of fact, the similar phenomena in the QCD case has been intensively investigated in recent years [28-41]. In the dilute limit, small $x$ gluons are almost fully linearly polarized just like the Weizsäcker-Williams photons. However, in the saturation regime, the linear polarization of small $x$ gluons will be affected by multiple rescattering effect and thus becomes process dependent. It has been proposed to probe the linearly polarized gluon distribution by measuring $\cos 2 \phi$ azimuthal asymmetries for two particle production in various high energy scattering processes at RHIC, LHC, or a future Electron-Ion Collider(EIC). Comparing the experimental measurements of gluon polarization and photon polarization in the small $x$ limit would be beneficial for studying saturation effect, which is absent in the QED case.

In our previous work [27], we addressed the azimuthal asymmetries for dilepton production with the equivalent photon approximation, which, however does not provide any information about the location of the particle production process. It does not allow us to distinguish UPC from central(or peripheral) collisions, as the impact parameter $\left(b_{\perp}\right)$ is implicitly integrated out in this method. The formalism for computing $b_{\perp}$ dependent cross section 
was developed in the early nineties of the past century in Refs. [42,43]. Calculations based on this more rigorous treatment, in some cases yield results significantly different from that computed in the equivalent photon approximation. For instance, theoretical calculations can not account for the measured lepton pair transverse momentum spectrum at low $q_{\perp}$ unless $b_{\perp}$ dependence is taken into account $[44,45]$.

The impact parameter dependent cross section for lepton pair production has been calculated only for the azimuthal angle averaged case in the aforementioned literatures. The purpose of the present work is to investigate the impact parameter dependence of the $\cos 4 \phi$ azimuthal asymmetry. The paper is structured as follows. In the next section, we derive the $b_{\perp}$ dependent polarized cross section for dilepton production in heavy ion collisions. We present the numerical estimations for the asymmetries in the central, peripheral, and ultraperipheral collisions at RHIC and LHC energy. The QED resummation effect is also included in our evaluations. The paper is summarized in Sec. III.

\section{THE IMPACT PARAMETER DEPENDENCE OF $\cos 4 \phi$ AZIMUTHAL ASYMMETRIES}

The dominant channel for dilepton production in the kinematical region where lepton pair transverse momentum is the order of the reverse of nucleus radius, is photonphoton fusion process,

$\gamma_{1}\left(x_{1} P+k_{1 \perp}\right)+\gamma_{2}\left(x_{2} \bar{P}+k_{2 \perp}\right) \rightarrow l^{+}\left(p_{1}\right)+l^{-}\left(p_{2}\right)$.

The leptons are produced nearly back-to-back in azimuthal with total transverse momentum $q_{\perp} \equiv p_{1 \perp}+p_{2 \perp}=k_{1 \perp}+k_{2 \perp}$ being much smaller than $P_{\perp}=\left(p_{1 \perp}-p_{2 \perp}\right) / 2$. When $P_{\perp}$ is sufficiently large, dilepton can be viewed as being produced locally. Since the location where dilepton is produced in the transverse plane is specified during our calculation, the incoming photons are no longer in the eigenstate of transverse momenta. To be more specific, the incoming photon $\gamma_{1}$ carries the different transverse momenta $k_{1 \perp}$ and $k_{1 \perp}^{\prime}$ in the amplitude and the conjugate amplitude respectively, while the transverse momenta of photon $\gamma_{2}$ are also different in the amplitude and the conjugate amplitude, and denoted as $k_{2 \perp}$ and $k_{2 \perp}^{\prime}$ respectively, with the constraint $k_{1 \perp}^{\prime}+k_{2 \perp}^{\prime} \equiv q_{\perp}$ [42].

Following the method outlined in Refs. [42,43], the impact parameter dependent cross section computed at the lowest order QED reads,

$$
\begin{aligned}
& \frac{d \sigma_{0}}{d^{2} p_{1 \perp} d^{2} p_{2 \perp} d y_{1} d y_{2} d^{2} b_{\perp}} \\
& =\frac{2 \alpha_{e}^{2}}{Q^{4}} \frac{1}{(2 \pi)^{2}}[\mathcal{A}+\mathcal{B} \cos 2 \phi+\mathcal{C} \cos 4 \phi]
\end{aligned}
$$

where $\phi$ is the angle between transverse momenta $q_{\perp}$ and $P_{\perp} \cdot y_{1}$ and $y_{2}$ are leptons rapidities, respectively. $b_{\perp}$ is the transverse distance between two colliding nuclei. $Q$ is the invariant mass of the lepton pair. The $\mathcal{B}$ term is proportional to the lepton mass and thus very small. We do not present the detailed expression for $\mathcal{B}$ here. Instead, we focus on investigating the $\cos 4 \phi$ asymmetry in this work which depends only on the $\mathcal{A}$ and $\mathcal{C}$ terms. The coefficients $\mathcal{A}$ and $\mathcal{C}$ take the form,

$$
\begin{aligned}
\mathcal{A}= & \frac{Q^{2}-2 P_{\perp}^{2}}{P_{\perp}^{2}} \frac{Z^{4} \alpha_{e}^{2}}{\pi^{4}} \int d^{2} k_{1 \perp} d^{2} k_{2 \perp} d^{2} \Delta_{\perp} \delta^{2}\left(q_{\perp}-k_{1 \perp}-k_{2 \perp}\right) e^{i \Delta_{\perp} \cdot b_{\perp}} \\
& \times\left[\left(k_{1 \perp} \cdot k_{1 \perp}^{\prime}\right)\left(k_{2 \perp} \cdot k_{2 \perp}^{\prime}\right)+\left(k_{1 \perp} \cdot k_{2 \perp}\right) \Delta_{\perp}^{2}-\left(k_{1 \perp} \cdot \Delta_{\perp}\right)\left(k_{2 \perp} \cdot \Delta_{\perp}\right)\right] \\
& \times \mathcal{F}\left(x_{1}, k_{1 \perp}^{2}\right) \mathcal{F}^{*}\left(x_{1}, k_{1 \perp}^{\prime}\right) \mathcal{F}\left(x_{2}, k_{2 \perp}^{2}\right) \mathcal{F}^{*}\left(x_{2}, k_{2 \perp}^{\prime}{ }^{2}\right)
\end{aligned}
$$

and

$$
\begin{aligned}
\mathcal{C}= & -2 \frac{Z^{4} \alpha_{e}^{2}}{\pi^{4}} \int d^{2} k_{1 \perp} d^{2} k_{2 \perp} d^{2} \Delta_{\perp} \delta^{2}\left(q_{\perp}-k_{1 \perp}-k_{2 \perp}\right) e^{i \Delta_{\perp} \cdot b_{\perp}} \\
& \times\left\{2\left[2\left(k_{2 \perp} \cdot \hat{q}_{\perp}\right)\left(k_{1 \perp} \cdot \hat{q}_{\perp}\right)-k_{1 \perp} \cdot k_{2 \perp}\right]\left[2\left(k_{2 \perp}^{\prime} \cdot \hat{q}_{\perp}\right)\left(k_{1 \perp}^{\prime} \cdot \hat{q}_{\perp}\right)-k_{1 \perp}^{\prime} \cdot k_{2 \perp}^{\prime}\right]\right. \\
& \left.-\left[\left(k_{1 \perp} \cdot k_{1 \perp}^{\prime}\right)\left(k_{2 \perp} \cdot k_{2 \perp}^{\prime}\right)+\left(k_{1 \perp} \cdot k_{2 \perp}\right) \Delta_{\perp}^{2}-\left(k_{1 \perp} \cdot \Delta_{\perp}\right)\left(k_{2 \perp} \cdot \Delta_{\perp}\right)\right]\right\} \\
& \times \mathcal{F}\left(x_{1}, k_{1 \perp}^{2}\right) \mathcal{F}^{*}\left(x_{1}, k_{1 \perp}^{\prime 2}\right) \mathcal{F}\left(x_{2}, k_{2 \perp}^{2}\right) \mathcal{F}^{*}\left(x_{2}, k_{2 \perp}^{\prime 2}\right)
\end{aligned}
$$

where $\Delta_{\perp}=k_{1 \perp}-k_{1 \perp}^{\prime}=k_{2 \perp}^{\prime}-k_{2 \perp} \cdot \hat{q}_{\perp}$ is unit vector defined as $\hat{q}_{\perp}=q_{\perp} /\left|q_{\perp}\right|$. The incoming photons longitudinal momenta fraction are fixed by the external kinematics according to $x_{1}=\sqrt{\frac{P_{\perp}^{2}+m^{2}}{s}}\left(e^{y_{1}}+e^{y_{2}}\right)$ and $x_{2}=\sqrt{\frac{P_{\perp}^{2}+m^{2}}{s}}\left(e^{-y_{1}}+e^{-y_{2}}\right)$ with $m$ being the lepton mass and $s$ being the center mass energy. The nuclear charge form factor enters the cross section via $\mathcal{F}\left(x, k_{\perp}^{2}\right)=\frac{F\left(k_{\perp}^{2}+x^{2} M_{p}^{2}\right)}{\left(k_{\perp}^{2}+x^{2} M_{p}^{2}\right)}$, where $M_{p}$ is proton mass. Note that the lepton mass is ignored in the hard coefficients. 
In comparison with our previous work where the calculation is formulated in TMD factorization, here we directly express the cross section as the convolutions of the form factor. Alternatively, one can factorize the impact parameter dependent cross section in terms of the photon Wigner distribution. However, this is beyond the scope of the present paper and will be addressed in a separate publication. Of course, if $b_{\perp}$ is integrated out in the above cross section formula, one can recover the cross section [27] derived using the equivalent photon approximation. This provides a nice consistency check. The $\cos 4 \phi$ azimuthal asymmetry is determined by the ratio between the terms $\mathcal{A}$ and $\mathcal{C}$. In general, the size of the asymmetry can only be numerically calculated because of the complicated convolutions involved. However, quite remarkably, the analytical solution is available at $b_{\perp}=0$. After a few steps of algebraic manipulations, one finds that the convolutions in $\mathcal{A}$ and $\mathcal{C}$ terms turn out to be identical for $b_{\perp}=0$. The asymmetry is then simply proportional to,

$$
\frac{\mathcal{C}\left(b_{\perp}=0\right)}{2 \mathcal{A}\left(b_{\perp}=0\right)}=\frac{-2 P_{\perp}^{2}}{2\left(Q^{2}-2 P_{\perp}^{2}\right)}
$$

which indicates that the $\cos 4 \phi$ asymmetry for pure electromagnetic lepton pair production in the central collisions is independent of $q_{\perp}$. This finding has been verified by the explicit numerical estimation as shown in Fig. 1. However, one should keep in mind that hadronic background contribution in central collisions is significant. The size of the measured asymmetry could be far below the predicated value.

In contrast to the most central collisions, strong interaction process is not necessarily the dominated source of lepton pair production in peripheral heavy ion collisions.

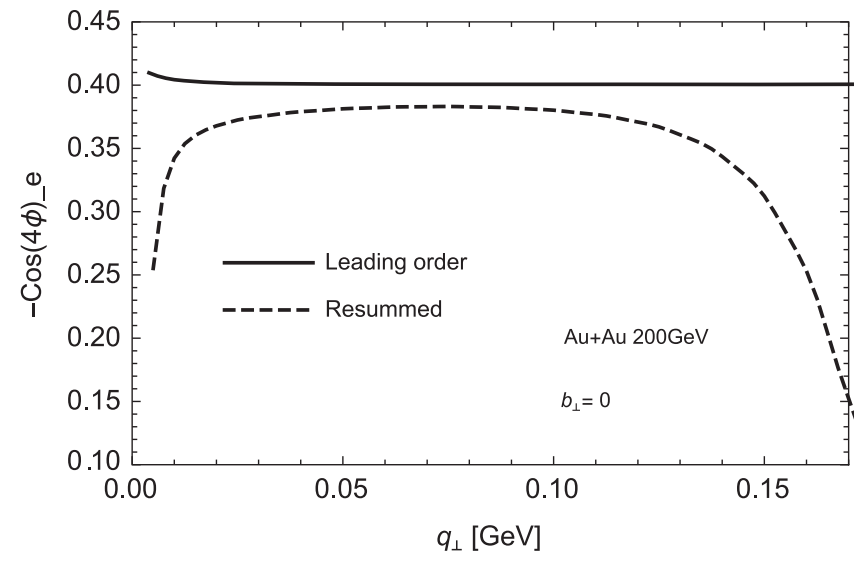

FIG. 1. The $\cos 4 \phi$ asymmetry in $\mathrm{Au}-\mathrm{Au}$ collisions at the impact parameter $b_{\perp}=0$ with and without taking into account resummation effect. The center mass energy is $\sqrt{s}=200 \mathrm{GeV}$. The electron and positron rapidities and transverse momenta are integrated over the regions $[-1,1]$, and $[0.2 \mathrm{GeV}, 0.4 \mathrm{GeV}]$ respectively.
Indeed, significant enhancements of lepton pair production at very low transverse momentum were observed by the STAR collaboration in peripheral hadronic Au-Au collisions [4]. This excess is a clear sign of coherent photonphoton interactions. A QED calculation using the coherent photon distribution essentially determined via the Coulomb law as the input turns out to describe experimental data extremely well at low pair transverse momentum for the various centrality classes [17]. This gives us confidence that the same treatment can be applied to compute azimuthal dependent cross section not only in UPCs but also in peripheral collisions.

We now proceed to describe the ingredients needed in the numerical evaluations of the asymmetry. First, the form factor is taken from the STARlight MC generator [24],

$F(|\vec{k}|)=\frac{4 \pi \rho^{0}}{|\vec{k}|^{3} A}\left[\sin \left(|\vec{k}| R_{A}\right)-|\vec{k}| R_{A} \cos \left(|\vec{k}| R_{A}\right)\right] \frac{1}{a^{2} \vec{k}^{2}+1}$

where $a=0.7 \mathrm{fm}$, and $\rho^{0}$ is a normalization factor. The nucleus radius is chosen to be $R_{A}=1.1 A^{1 / 3} \mathrm{fm}$ for $\mathrm{Au}$ and $\mathrm{Pb}$ targets, $R_{A}=1.2 A^{1 / 3}$ fm for Ru target. This parametrization is very close to the Woods-Saxon distribution, and is used in our numerical evaluation. In order to take into account the effect of final state multiple soft photon radiation, a Sudakov factor has to be inserted in the differential cross section in $r_{\perp}$ space,

$$
\begin{aligned}
& \frac{d \sigma}{d^{2} p_{1 \perp} d^{2} p_{2 \perp} d y_{1} d y_{2} d^{2} b_{\perp}} \\
& =\int \frac{d^{2} r_{\perp}}{(2 \pi)^{2}} e^{i r_{\perp} \cdot q_{\perp}} e^{-S\left(Q, r_{\perp}\right)} \int d^{2} q_{\perp}^{\prime} e^{i r_{\perp} \cdot q_{\perp}^{\prime}} d \sigma_{0}\left(q_{\perp}^{\prime}, \ldots\right)
\end{aligned}
$$

where the Sudakov factor at one loop is given by [16],

$$
S\left(Q, r_{\perp}\right)= \begin{cases}\frac{\alpha_{e}}{2 \pi} \ln ^{2} \frac{Q^{2}}{\mu_{r}^{2}}, & \mu_{r}>m_{\mu} \\ \frac{\alpha_{e}}{2 \pi} \ln \frac{Q^{2}}{m_{\mu}^{2}}\left[\ln \frac{Q^{2}}{\mu_{r}^{2}}+\ln \frac{m_{\mu}^{2}}{\mu_{r}^{2}}\right], & \mu_{r}<m_{\mu}\end{cases}
$$

with $\mu_{r}=2 e^{-\gamma_{E}} /\left|r_{\perp}\right|$. The perturbative tail at relatively high $q_{\perp}$ is mainly generated by this Sudakov factor. The theoretical calculation is in good agreement with the ATLAS UPC high $q_{\perp}$ data [16]. In contrast, at intermediate lepton pair transverse momentum, roughly speaking $10 \mathrm{MeV}<q_{\perp}<70 \mathrm{MeV}$, the $q_{\perp}$ shape is determined by the primordial photon distribution. At low $q_{\perp}(<10 \mathrm{MeV})$, the smallness of the fine coupling constant is compensated by the large double logarithm $\ln ^{2} \frac{Q^{2}}{\mu_{b}^{2}}$, leading to a significant resummation effect, which, for example, has been clearly demonstrated in Fig. 1.

The numerical results for the computed azimuthal asymmetries for the different collisions species and 

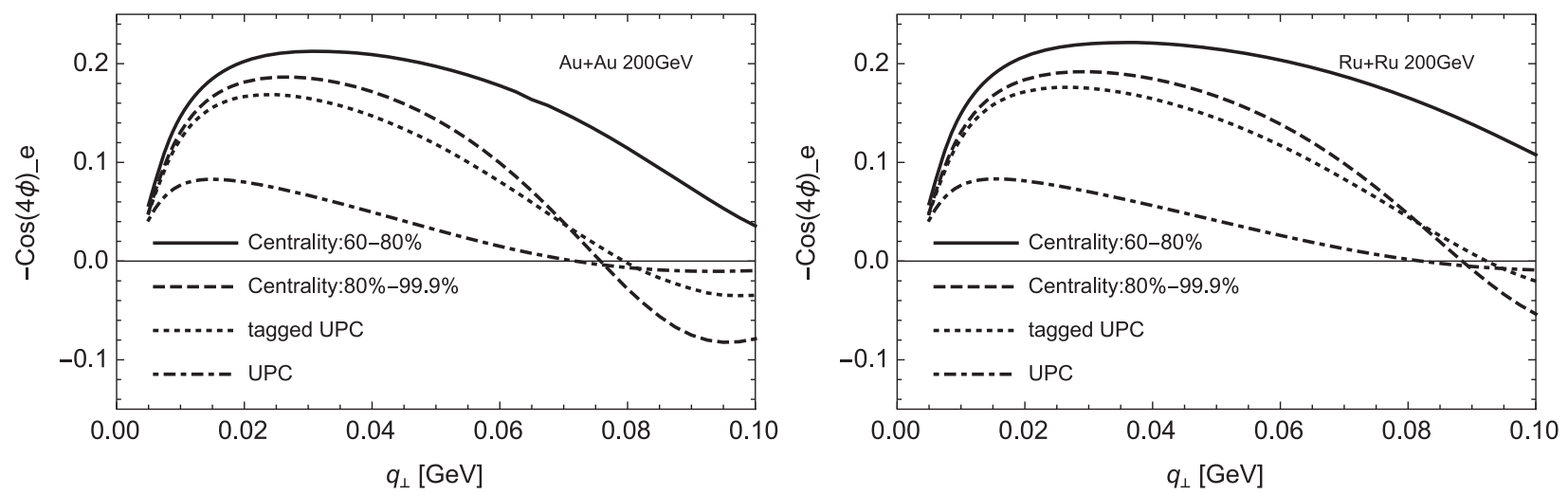

FIG. 2. Estimates of the $\cos 4 \phi$ asymmetry as the function of $q_{\perp}$ for the different centralities at $\sqrt{s}=200 \mathrm{GeV}$. The electron and positron rapidities and transverse momenta are integrated over the regions $[-1,1]$, and $[0.2 \mathrm{GeV}, 0.4 \mathrm{GeV}]$. The asymmetries in Au-Au collisions and $\mathrm{Ru}-\mathrm{Ru}$ collisions are shown in the left plot and the right plot respectively.

centralities are presented in Figs. 2 and 3. Here the azimuthal asymmetries, i.e., the average value of $\cos 4 \phi$ are defined as,

$$
\langle\cos (4 \phi)\rangle=\frac{\int \frac{d \sigma}{d \mathcal{P} . S .} \cos 4 \phi d \mathcal{P} . S .}{\int \frac{d \sigma}{d \mathcal{P} . S .} d \mathcal{P} . S .}
$$

We compute the asymmetry for two deferent centrality classes as well as for the UPC and the tagged UPC cases. The corresponding impact parameter range for a given centrality class is determined using the Glauber model(see the review article [46] and references therein). For the UPC, the asymmetry is averaged over the impact parameter range $\left[2 R_{A}, \infty\right]$. However, STAR experiments at RHIC measure pair production cross section together with the double electromagnetic excitation in both ions. Neutrons emitted at forward angles by the fragmenting nuclei are measured, and used as a UPC trigger. Requiring lepton pair to be produced in coincidence with Coulomb breakup of the

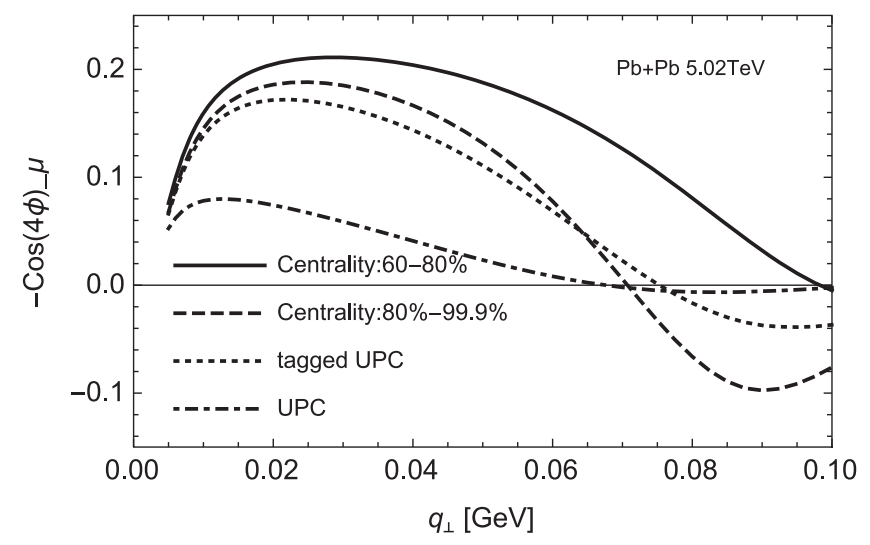

FIG. 3. Estimates of the $\cos 4 \phi$ asymmetry as the function of $q_{\perp}$ for the different centralities at $\sqrt{s}=5020 \mathrm{GeV}$. The muon and antimuon rapidities and transverse momenta are integrated over the regions $[-1,1]$, and $[4 \mathrm{GeV}, 45 \mathrm{GeV}]$, respectively. beam nuclei alters the impact parameter distribution compared with exclusive production. In order to incorporate the experimental conditions in the theoretical calculations, one can define a "tagged" UPC cross section,

$$
2 \pi \int_{2 R_{A}}^{\infty} b_{\perp} d b_{\perp} P^{2}\left(b_{\perp}\right) d \sigma\left(b_{\perp}, \ldots\right)
$$

where the probability $P\left(b_{\perp}\right)$ of emitting a neutron from the scattered nucleus is often parametrized as [47],

$$
\begin{aligned}
P\left(b_{\perp}\right)= & 5.45 * 10^{-5} \frac{Z^{3}(A-Z)}{A^{2 / 3} b_{\perp}^{2}} \\
& \times \exp \left[-5.45 * 10^{-5} \frac{Z^{3}(A-Z)}{A^{2 / 3} b_{\perp}^{2}}\right] .
\end{aligned}
$$

As a matter of fact, the mean impact parameter is dramatically reduced in interactions with Coulomb dissociation.

We plot the $\cos 4 \phi$ asymmetry for electron pair production at midrapidity as the function of the total transverse momentum $q_{\perp}$ at the center mass energy $\sqrt{s}=200 \mathrm{GeV}$ in Fig. 2. The general trend is that the asymmetry increases when the impact parameter decreases. The overall $q_{\perp}$ and $b_{\perp}$ dependent behavior of the asymmetry for the different collision species( $\mathrm{Au}$ and $\mathrm{Ru}$ ) are similar, except for that the curves are slightly more flat for the smaller nucleus. The asymmetry reaches a maximal value of $17 \%-22 \%$ percent around $q_{\perp} \approx 30 \mathrm{MeV}$ for the centrality classes [60\%-80\%], [80\%-99.9\%], and the tagged UPC. For the unrestricted UPC, the asymmetry is roughly twice smaller than that in the tagged UPC. The results obtained for dimuon production in $\mathrm{Pb}-\mathrm{Pb}$ collisions at LHC energy shown in Fig. 3 are rather close to these at RHIC energy.

\section{CONCLUSIONS}

We study the impact parameter dependence of the $\cos 4 \phi$ azimuthal asymmetry for purely electromagnetic lepton 
pair production in heavy ion collisions at low $q_{\perp}$. This asymmetry arises from the correlation between the polarization vector of the electric field coherently generated by a fast moving heavy ion and the associated equivalent photon's transverse momentum. Such correlation reflects the nature of the boosted Coulomb potential. We found that the azimuthal asymmetry has a strong $b_{\perp}$ dependence. To be more specific, the asymmetry decreases with increasing impact parameter. Moreover, the $q_{\perp}$ dependent behavior of the azimuthal asymmetry is different in the different $b_{\perp}$ regions. We present numerical results for the $b_{\perp}$ and $q_{\perp}$ dependent asymmetry for the different collision species at various center mass energies. It would be interesting to test these theoretical predications at RHIC and LHC.

The study of such initial state effect in heavy ion collisions is not only important for facilitating the investigations of the electromagnetic properties of QGP, but also interesting in its own right. For instance, the polarization dependent observable can be used as a powerful tool to study QED processes in strong electromagnetic fields, particularly in view of the fact that no definitive conclusion on Coulomb correction has yet been drawn on experimental side. It has an advantage over the azimuthal angle averaged cross section in this regard because the absolute normalization which suffers various uncertainties are cancelled out when computing the asymmetry. Furthermore, comparing photon's linear polarization and gluon's linear polarization in the small $x$ limit would be helpful for us to gain more insight into how the gluon polarization is affected by saturation effect.

\section{ACKNOWLEDGMENTS}

We thank Zhang-bu Xu, Wang-mei Zha, and James Daniel Brandenburg for drawing our attention to the Ref. [42]. J. Zhou thanks Feng Yuan, Bowen Xiao, and Chi Yang for helpful discussions. J. Zhou has been supported by the National Science Foundations of China under Grant No. 11675093, and by the Thousand Talents Plan for Young Professionals. Ya-jin Zhou has been supported by the National Science Foundations of China under Grant No. 11675092.
[1] G. Agakichiev et al. (CERES Collaboration), Phys. Rev. Lett. 75, 1272 (1995).

[2] R. Arnaldi et al. (NA60 Collaboration), Phys. Rev. Lett. 96, 162302 (2006).

[3] J. Adams et al. (STAR Collaboration), Phys. Rev. C 70, 031902 (2004).

[4] J. Adam et al. (STAR Collaboration), Phys. Rev. Lett. 121, 132301 (2018).

[5] J. Adam et al. (STAR Collaboration), arXiv:1810.10159.

[6] M. Aaboud et al. (ATLAS Collaboration), Phys. Rev. Lett. 121, 212301 (2018).

[7] S. Acharya et al. (ALICE Collaboration), Phys. Rev. C 99, 024002 (2019).

[8] H. A. Bethe and L. C. Maximon, Phys. Rev. 93, 768 (1954).

[9] H. Davies, H. A. Bethe, and L. C. Maximon, Phys. Rev. 93, 788 (1954).

[10] B. Segev and J. C. Wells, Phys. Rev. A 57, 1849 (1998).

[11] D. Ivanov and K. Melnikov, Phys. Rev. D 57, 4025 (1998).

[12] A. J. Baltz, F. Gelis, L. D. McLerran, and A. Peshier, Nucl. Phys. A695, 395 (2001).

[13] U. Eichmann, J. Reinhardt, and W. Greiner, Phys. Rev. A 59, 1223 (1999).

[14] A. J. Baltz, Phys. Rev. Lett. 100, 062302 (2008).

[15] W. Zha, L. Ruan, Z. Tang, Z. Xu, and S. Yang, Phys. Lett. B 781, 182 (2018).

[16] S. Klein, A. H. Mueller, B. W. Xiao, and F. Yuan, Phys. Rev. Lett. 122, 132301 (2019).

[17] W. Zha, J. D. Brandenburg, Z. Tang, and Z. Xu, Phys. Lett. B 800, 135089 (2020).

[18] S. R. Klein, Phys. Rev. C 97, 054903 (2018).
[19] Y. J. Ye, Y. G. Ma, A. H. Tang, and G. Wang, Phys. Rev. C 99, 044901 (2019).

[20] C. A. Bertulani and G. Baur, Phys. Rep. 163, 299 (1988).

[21] C. A. Bertulani, S. R. Klein, and J. Nystrand, Annu. Rev. Nucl. Part. Sci. 55, 271 (2005).

[22] G. Baur, K. Hencken, and D. Trautmann, Phys. Rep. 453, 1 (2007).

[23] A. J. Baltz, G. Baur, D. Denterria, L. Frankfurt, F. Gelis, V. Guzey, K. Hencken, Y. Kharlov, M. Klasen, and S. Klein, Phys. Rep. 458, 1 (2008).

[24] S. R. Klein, J. Nystrand, J. Seger, Y. Gorbunov, and J. Butterworth, Comput. Phys. Commun. 212, 258 (2017).

[25] Z. L. Ma and J. Q. Zhu, Phys. Rev. D 97, 054030 (2018).

[26] P. J. Mulders and J. Rodrigues, Phys. Rev. D 63, 094021 (2001).

[27] C. Li, J. Zhou, and Y. J. Zhou, Phys. Lett. B 795, 576 (2019).

[28] C. Pisano, D. Boer, S. J. Brodsky, M. G. A. Buffing, and P. J. Mulders, J. High Energy Phys. 10 (2013) 024.

[29] A. Metz and J. Zhou, Phys. Rev. D 84, 051503 (2011).

[30] F. Dominguez, J. W. Qiu, B. W. Xiao, and F. Yuan, Phys. Rev. D 85, 045003 (2012).

[31] D. Boer, P. J. Mulders, and C. Pisano, Phys. Rev. D 80, 094017 (2009).

[32] D. Boer, S. J. Brodsky, P. J. Mulders, and C. Pisano, Phys. Rev. Lett. 106, 132001 (2011).

[33] J.-W. Qiu, M. Schlegel, and W. Vogelsang, Phys. Rev. Lett. 107, 062001 (2011).

[34] A. Schafer and J. Zhou, Phys. Rev. D 85, 114004 (2012).

[35] E. Akcakaya, A. Schäfer, and J. Zhou, Phys. Rev. D 87, 054010 (2013). 
[36] A. Dumitru, T. Lappi, and V. Skokov, Phys. Rev. Lett. 115, 252301 (2015).

[37] P. Kotko, K. Kutak, C. Marquet, E. Petreska, S. Sapeta, and A. van Hameren, J. High Energy Phys. 09 (2015) 106.

[38] D. Boer, P. J. Mulders, J. Zhou, and Y. J. Zhou, J. High Energy Phys. 10 (2017) 196.

[39] C. Marquet, C. Roiesnel, and P. Taels, Phys. Rev. D 97, 014004 (2018).

[40] D. Gutierrez-Reyes, S. Leal-Gomez, I. Scimemi, and A. Vladimirov, J. High Energy Phys. 11 (2019) 121.

[41] F. Scarpa, D. Boer, M. G. Echevarria, J. P. Lansberg, C. Pisano, and M. Schlegel, arXiv:1909.05769.
[42] M. Vidovic, M. Greiner, C. Best, and G. Soff, Phys. Rev. C 47, 2308 (1993).

[43] K. Hencken, D. Trautmann, and G. Baur, Phys. Rev. A 49, 1584 (1994); 51, 1874 (1995).

[44] K. Hencken, G. Baur, and D. Trautmann, Phys. Rev. C 69, 054902 (2004).

[45] A. J. Baltz, Phys. Rev. C 74, 054903 (2006).

[46] M. L. Miller, K. Reygers, S. J. Sanders, and P. Steinberg, Annu. Rev. Nucl. Part. Sci. 57, 205 (2007).

[47] G. Baur, K. Hencken, and D. Trautmann, J. Phys. G 24, 1657 (1998). 